\title{
FINITE ELEMENT DISCRETIZATION OF SEMILINEAR ACOUSTIC WAVE EQUATIONS WITH KINETIC BOUNDARY CONDITIONS*
}

\author{
MARLIS HOCHBRUCK ${ }^{\dagger}$ AND JAN LEIBOLD ${ }^{\dagger}$
}

\begin{abstract}
We consider isoparametric finite element discretizations of semilinear acoustic wave equations with kinetic boundary conditions and derive a corresponding error bound as our main result. The difficulty is that such problems are stated on domains with curved boundaries and this renders the discretizations nonconforming. Our approach is to provide a unified error analysis for nonconforming space discretizations for semilinear wave equations. In particular, we introduce a general, abstract framework for nonconforming space discretizations in which we derive a-priori error bounds in terms of interpolation, data, and conformity errors. The theory applies to a large class of problems and discretizations that fit into the abstract framework. The error bound for wave equations with kinetic boundary conditions is obtained from the general theory by inserting known interpolation and geometric error bounds into the abstract error result of the unified error analysis.
\end{abstract}

Key words. wave equation, dynamic boundary conditions, nonconforming space discretization, error analysis, a-priori error bounds, semilinear evolution equations, operator semigroups, isoparametric finite elements

AMS subject classifications. 65M12, 65M15, 65J08, 65M60

1. Introduction. The aim of this paper is to introduce and analyze finite element discretizations of semilinear acoustic wave equations with kinetic boundary conditions. Kinetic boundary conditions serve as an effective model for the interaction of waves with obstacles or boundaries that are covered by materials with distinctive elastic or damping properties, where the wave length is large compared to the width of the boundary layer; see, e.g., [14, Section 3.2]. We refer to $[16,17]$ and references therein for more information and analytical results about these equations.

Kinetic boundary conditions are a special case of dynamic boundary conditions that contain tangential derivatives and are intrinsically posed on domains with (piecewise) smooth and therefore possibly curved boundaries. Hence, most methods are applied on an approximated domain rendering the approximation nonconforming. This makes the error analysis much more involved. Such problems were addressed in [7, 8], where a unified error analysis (UEA) was introduced that allows to analyze nonconforming space discretizations of linear wave equations in a systematic way. The UEA yields an abstract error result that can be used to prove convergence rates for specific equations and discretizations using geometric and interpolation error results. Finite element discretizations of linear wave equations with linear kinetic boundary conditions are only specific examples fitting into the abstract framework. Several others are discussed in $[7,8]$.

While working on our main goal, namely to provide a rigorous error analysis for finite element discretizations of semilinear wave equations with kinetic boundary conditions, it turned out that major parts of the theoretical investigations hold in a much more general framework. Hence, we first extend the UEA to a large class of semilinear evolution equations. The main difficulty in discretizing and analyzing semilinear problems compared to linear ones is the discretization of the nonlinear term. This has to be done in such a way that the discretization preserves the Lipschitz continuity of the nonlinearity with a Lipschitz constant that is independent of the underlying mesh. Additionally, it has to be shown that the

\footnotetext{
${ }^{*}$ Received December 23, 2019. Accepted June 29, 2020. Published online on August 19, 2020. Recommended by Marco Donatelli.

${ }^{\dagger}$ Karlsruhe Institute of Technology, Institute for Applied and Numerical Mathematics, Englerstraße 2, D-76131 Karlsruhe (\{marlis.hochbruck, jan.leibold\}@kit.edu).
} 
discretization error has the correct order of convergence. Our abstract error result leads to convergence rates of order $p$ for order- $p$ finite elements.

The extension of the UEA to semilinear problem allows us not only to treat the specific application we are interested in, but it also applies to several other applications; cf. [8] for details. These applications range from conformal discretizations of acoustic wave equations to heterogeneous multiscale methods applied to Maxwell's equations; cf. [10]. In fact, with our new results it is straightforward to treat these applications also in the presence of semilinear perturbations if the nonlinear part and its spatial discretization satisfy a Lipschitz condition. We thus do not investigate this further in the current paper.

As in [7, 8], we derive the error bounds in an energy norm. Subsequently, $L^{2}$-error bounds for different types of dynamic boundary conditions for linear problems were proven in [9]. The analysis is quite involved and requires different techniques. Therefore, generalizing it to semilinear problems is beyond the scope of this paper.

To the best of our knowledge this is the first error analysis for semilinear wave equations with kinetic boundary conditions. In [12] semilinear parabolic problems with dynamic boundary conditions were analyzed, but the techniques do not apply to the hyperbolic case.

The paper is organized as follows: in Section 2 we introduce semilinear acoustic wave equations with kinetic boundary conditions and their space discretizations with isoparametric finite elements. Furthermore, we state the main result of the paper, namely a space discretization error bound of order $p$ in the energy norm for order- $p$ isoparametric elements. In Section 3 we present the unified error analysis and prove abstract error bounds for nonconforming space discretizations of semilinear evolution equations. These bounds are used in Section 4 to provide error estimates for the discretizations of semilinear wave equations with kinetic boundary conditions. Finally, in Section 5 we conclude with a numerical experiment.

2. Wave equations with kinetic boundary conditions: problem statement. In this section we introduce wave equations with kinetic boundary conditions. After formulating the equations in a suitable analytical setting, we present a finite element space discretization and the main error result that will be proven in Section 4. Wave equations with kinetic boundary conditions were already studied in [7] in the linear case.

2.1. Formulation of the equations. Let $\Omega \subset \mathbb{R}^{d}, d=2,3$, be a bounded domain with $C^{2}$ boundary $\Gamma=\partial \Omega$. With $\Delta_{\Gamma}$ we denote the Laplace-Beltrami operator on $\Gamma$ and with $n$ the outer normal vector. For semilinear wave equations with kinetic boundary conditions, we seek $u:[0, T] \times \bar{\Omega} \rightarrow \mathbb{R}$ satisfying

$$
\left\{\begin{aligned}
u_{t t}+\left(\alpha_{\Omega}+\beta_{\Omega} \cdot \nabla\right) u_{t}-\Delta u & =\widetilde{f}_{\Omega}(t, \mathbf{x}, u), & & \text { in }(0, T) \times \Omega, \\
u_{t t}+\partial_{n} u+\left(\alpha_{\Gamma}+\beta_{\Gamma} \cdot \nabla_{\Gamma}\right) u_{t}-\Delta_{\Gamma} u & =\widetilde{f}_{\Gamma}(t, \mathbf{x}, u), & & \text { in }(0, T) \times \partial \Omega, \\
u(0, \mathbf{x})=u^{0}(\mathbf{x}), & u_{t}(0, \mathbf{x})=v^{0}(\mathbf{x}), & & \text { in } \bar{\Omega} .
\end{aligned}\right.
$$

We assume $T<t^{*}\left(u^{0}, v^{0}\right)$, where $t^{*}\left(u^{0}, v^{0}\right)$ denotes the maximal existence time of the (weak) solution of (2.1).

More general problems, for instance, containing additional material parameters, can be found in [7]. For the sake of presentation, we omit these more general problems here and focus on the additional difficulties caused by the nonlinearity.

We require the following assumptions throughout the rest of the paper:

ASSUMPTION 2.1.

(a) The nonlinearities satisfy

(i) $\widetilde{f}_{\Omega} \in C^{1}([0, T] \times \bar{\Omega} \times \mathbb{R} ; \mathbb{R})$,

(ii) $\widetilde{f}_{\Gamma} \in C^{1}([0, T] \times \Gamma \times \mathbb{R} ; \mathbb{R})$, 
and the following growth condition: there exist

$$
\zeta_{\Omega}\left\{\begin{array} { l l } 
{ < \infty , } & { d = 2 , } \\
{ \leq \frac { d } { d - 2 } , } & { d \geq 3 , }
\end{array} \quad \text { and } \quad \zeta _ { \Gamma } \left\{\begin{array}{ll}
<\infty, & d=2,3, \\
\leq \frac{d-1}{d-3}, & d \geq 4,
\end{array}\right.\right.
$$

such that for all $(t, \mathbf{x}, u) \in[0, T] \times \Omega \times \mathbb{R}$

$$
\begin{aligned}
\left|\widetilde{f}_{\Omega}(t, \mathbf{x}, u)\right| & \leq C\left(1+|u|^{\zeta_{\Omega}}\right), \\
\left|\nabla \widetilde{f}_{\Omega}(t, \mathbf{x}, u)\right| & \leq C\left(1+|u|^{\zeta_{\Omega}-1}\right),
\end{aligned}
$$

and for all $(t, \mathbf{x}, u) \in[0, T] \times \Gamma \times \mathbb{R}$

$$
\begin{aligned}
\left|\widetilde{f}_{\Gamma}(t, \mathbf{x}, u)\right| & \leq C\left(1+|u|^{\zeta_{\Gamma}}\right), \\
\left|\nabla \widetilde{f}_{\Gamma}(t, \mathbf{x}, u)\right| & \leq C\left(1+|u|^{\zeta_{\Gamma}-1}\right)
\end{aligned}
$$

hold true.

(b) The coefficients $\alpha_{\Omega} \in C(\bar{\Omega}), \beta_{\Omega} \in C^{1}(\bar{\Omega})^{d}, \alpha_{\Gamma} \in C(\Gamma)$, and $\beta_{\Gamma} \in C^{1}(\Gamma)^{d}$ are non-negative and satisfy

$$
\alpha_{\Omega}-\frac{1}{2} \operatorname{div} \beta_{\Omega} \geq 0 \quad \text { in } \Omega, \quad \alpha_{\Gamma}+\frac{1}{2}\left(\beta_{\Omega} \cdot n-\operatorname{div}_{\Gamma} \beta_{\Gamma}\right) \geq 0 \quad \text { on } \Gamma .
$$

Because of (2.2) we have by the Sobolev embedding theorem, cf., e.g., [1, Theorem 4.12]

$$
H^{1}(\Omega) \hookrightarrow L^{2 \zeta_{\Omega}}(\Omega) \quad \text { and } \quad H^{1}(\Gamma) \hookrightarrow L^{2 \zeta_{\Gamma}}(\Gamma) .
$$

We continue by presenting the weak formulation of (2.1). For this we define

$$
H:=L^{2}(\Omega) \times L^{2}(\Gamma) \quad \text { and } \quad V:=H^{1}(\Omega ; \Gamma),
$$

where

$$
H^{k}(\Omega ; \Gamma):=\left\{v \in H^{k}(\Omega) \mid \gamma(v) \in H^{k}(\Gamma)\right\}, \quad k \geq 1,
$$

and $\gamma$ denotes the trace operator. For more information on surface Sobolev spaces we refer to [6]. Further, we define the bilinear forms $m: H \times H \rightarrow \mathbb{R}, b: V \times H \rightarrow \mathbb{R}$, and $a: V \times V \rightarrow \mathbb{R}$ via

$$
\begin{aligned}
m(v, \varphi) & =\int_{\Omega} v \varphi \mathrm{d} \mathbf{x}+\int_{\Gamma} v \varphi \mathrm{d} s \\
b(v, \varphi) & =\int_{\Omega}\left(\alpha_{\Omega} v+\beta_{\Omega} \cdot \nabla v\right) \varphi \mathrm{d} \mathbf{x}+\int_{\Gamma}\left(\alpha_{\Gamma} v+\beta_{\Gamma} \cdot \nabla_{\Gamma} v\right) \varphi \mathrm{d} s \\
a(v, \varphi) & =\int_{\Omega} \nabla v \cdot \nabla \varphi \mathrm{d} \mathbf{x}+\int_{\Gamma} \nabla_{\Gamma} v \cdot \nabla_{\Gamma} \varphi \mathrm{d} s
\end{aligned}
$$

and the nonlinear function $f:[0, T] \times V \rightarrow H$ via

$$
m(f(t, v), \varphi)=\int_{\Omega}\left(\widetilde{f}_{\Omega}(t, \cdot, v(\cdot))\right) \varphi \mathrm{d} \mathbf{x}+\int_{\Gamma}\left(\widetilde{f}_{\Gamma}(t, \cdot, v(\cdot))\right) \varphi \mathrm{d} s .
$$

We have that $H$ is a Hilbert space with scalar product $m$, and it can be proven that $V$ is a Hilbert space with scalar product $\widetilde{a}:=a+m$ (cf. [11, Lemma 2.5]), which is densely embedded in $H$ via

$$
V \ni v \mapsto(v, \gamma(v)) \in H^{1}(\Omega) \times H^{1}(\Gamma) \subset H
$$


The weak formulation of (2.1) is a special case of the more general variational problem: seek $u \in C^{2}([0, T] ; H) \cap C^{1}([0, T] ; V)$ such that

$$
\begin{aligned}
m\left(u^{\prime \prime}, \varphi\right)+b\left(u^{\prime}, \varphi\right)+a(u, \varphi) & =m\left(f\left(t, u, u^{\prime}\right), \varphi\right), \\
u(0)=u^{0}, \quad u^{\prime}(0) & =v^{0},
\end{aligned}
$$

for all $\varphi \in V$ and $t \in(0, T]$.

Note that for the specific application (2.1), the nonlinearity $f$ defined in (2.6) does not depend on $u^{\prime}$, but the general framework that we consider covers this case. The bilinear forms and the nonlinearity satisfy the following general assumption.

Assumption 2.2.

(a) The bilinear form $m$ is a scalar product on $H$ with induced norm $\|\cdot\|_{m}$.

(b) $a: V \times V \rightarrow \mathbb{R}$ is a symmetric bilinear form and there exists a constant $c_{G} \geq 0$ such that

$$
\widetilde{a}:=a+c_{G} m
$$

is a scalar product on $V$ with induced norm $\|\cdot\|_{\widetilde{a}}$.

(c) The bilinear form $b: V \times H \rightarrow \mathbb{R}$ is continuous, and there exists $\beta_{\mathrm{qm}} \geq 0$ such that

$$
b(v, v)+\beta_{\mathrm{qm}}\|v\|_{m}^{2} \geq 0 \quad \text { for all } v \in V .
$$

(d) The nonlinearity $f$ satisfies $f \in C^{1}([0, T] \times V \times H ; H)$ and is locally Lipschitzcontinuous on $V \times H$ with Lipschitz-constant $L_{T, M}$, i.e., for all $t \in[0, T]$ and $x=[v, w], y=[\widehat{v}, \widehat{w}] \in V \times H$ with $\|x\|_{V \times H},\|y\|_{V \times H} \leq M:$

$$
\|f(t, v, w)-f(t, \widehat{v}, \widehat{w})\|_{X} \leq L_{T, M}\|x-y\|_{V \times H}
$$

In [7] it was shown that for the bilinear forms defined in (2.5) we have $c_{G}=1$ and $\beta_{\mathrm{qm}}=0$. The differentiability and Lipschitz-continuity of $f$ was proven in [13, Lemma 4.2]; more general results can be found in [5]. We will see in Section 4 that under Assumption 2.2, problem (2.7) is (locally) well-posed.

2.2. Space discretization. To discretize (2.1) in space, we use the bulk-surface finite element method presented in [4]. This discretization was also considered in [7] for linear problems. The additional difficulty here is the discretization of the nonlinearity.

We start by giving a short summary of the bulk-surface finite element method; cf. [4, 7] for more details.

Bulk-surface finite element method. Let $\mathcal{T}_{h}$ be a consistent quasi-uniform mesh of isoparametric elements $K$ of degree $p$ with mesh width $h$. The discretized domain and its boundary are denoted by

$$
\Omega_{h}:=\bigcup_{K \in \mathcal{T}_{h}} K \approx \Omega \quad \text { and } \quad \Gamma_{h}:=\partial \Omega_{h} .
$$

We define the bulk and the surface finite element space of order $p \geq 1$ via

$$
\begin{aligned}
V_{h, p}^{\Omega} & :=\left\{v_{h} \in C\left(\Omega_{h}\right)\left|v_{h}\right|_{K}=\widehat{v}_{h} \circ\left(F_{K}\right)^{-1} \text { with } \widehat{v}_{h} \in \mathbb{P}_{p}(\widehat{K}) \text { for all } K \in \mathcal{T}_{h}\right\}, \\
V_{h, p}^{\Gamma} & :=\left\{\vartheta_{h} \in C\left(\Gamma_{h}\right)\left|\vartheta_{h}=v_{h}\right|_{\Gamma_{h}} \text { with } v_{h} \in V_{h, p}^{\Omega}\right\} .
\end{aligned}
$$

Here $\mathbb{P}_{p}(\widehat{K})$ denotes the space of polynomial of degree $p$ on a reference triangle $\widehat{K}$, and $F_{K}$ is a transformation from $\widehat{K}$ to $K$. Since this discretization is nonconforming due to $\Omega_{h} \neq \Omega$, we 
need a lift operator to relate the analytical and the numerical solution. In [4] an element-wise smooth homeomorphism $G_{h}: \Omega_{h} \rightarrow \Omega$ with

$$
\left.G_{h}\right|_{K} \in C^{p+1}(K), \quad \text { for all } p \leq k \text { and } K \in \mathcal{T}_{h},
$$

is constructed. This allows us to define lifted versions of $v_{h} \in V_{h, p}^{\Omega}$ and $\vartheta_{h} \in V_{h, p}^{\Gamma}$ as

$$
v_{h}^{\ell}:=v_{h} \circ G_{h}^{-1} \quad \text { and } \quad \vartheta_{h}^{\ell} \quad:=\vartheta_{h} \circ G_{h}^{-1} .
$$

The mapping $G_{h}$ is constructed in such a way that $G_{h}\left(a_{i}\right)=a_{i}, i=1, \ldots, N=\operatorname{dim} V_{h}$, where $a_{1}, \ldots, a_{N} \in \Omega_{h}$ are the nodes corresponding to the finite element discretization. This implies $v_{h}^{\ell}\left(a_{i}\right)=v_{h}\left(a_{i}\right)$ for $i=1, \ldots, N$ and for all $v_{h} \in V_{h, p}^{\Omega}$. Furthermore, it was shown in [4] that there exist constants $c_{\Omega, \Omega_{h}}, c_{\Gamma, \Gamma_{h}}, C_{\Omega, \Omega_{h}}, C_{\Gamma, \Gamma_{h}}>0$ independent of $h$ such that for all $v_{h} \in V_{h, p}^{\Omega}, \vartheta_{h} \in V_{h, p}^{\Gamma}$ the following norm equivalences

$$
\begin{aligned}
& c_{\Omega, \Omega_{h}}\left\|v_{h}\right\|_{L^{2}\left(\Omega_{h}\right)} \leq\left\|v_{h}^{\ell}\right\|_{L^{2}(\Omega)} \leq C_{\Omega, \Omega_{h}}\left\|v_{h}\right\|_{L^{2}\left(\Omega_{h}\right)}, \\
& c_{\Omega, \Omega_{h}}\left\|\nabla v_{h}\right\|_{L^{2}\left(\Omega_{h}\right)} \leq\left\|\nabla v_{h}^{\ell}\right\|_{L^{2}(\Omega)} \leq C_{\Omega, \Omega_{h}}\left\|\nabla v_{h}\right\|_{L^{2}\left(\Omega_{h}\right)}, \\
& c_{\Gamma, \Gamma_{h}}\left\|\vartheta_{h}\right\|_{L^{2}\left(\Gamma_{h}\right)} \leq\left\|\vartheta_{h}^{\ell}\right\|_{L^{2}(\Gamma)} \leq C_{\Gamma, \Gamma_{h}}\left\|\vartheta_{h}\right\|_{L^{2}\left(\Gamma_{h}\right)}, \\
& c_{\Gamma, \Gamma_{h}}\left\|\nabla_{\Gamma} \vartheta_{h}\right\|_{L^{2}\left(\Gamma_{h}\right)} \leq\left\|\nabla_{\Gamma} \vartheta_{h}^{\ell}\right\|_{L^{2}(\Gamma)} \leq C_{\Gamma, \Gamma_{h}}\left\|\nabla_{\Gamma} \vartheta_{h}\right\|_{L^{2}\left(\Gamma_{h}\right)}
\end{aligned}
$$

holds true.

With $I_{h, \Omega}: C(\bar{\Omega}) \rightarrow V_{h, p}^{\Omega}$ and $I_{h, \Gamma}: C(\Gamma) \rightarrow V_{h, p}^{\Gamma}$ we denote the order $p$ nodal interpolation operator in $\Omega$ and on $\Gamma$, respectively. The interpolation operators satisfy

$$
\begin{aligned}
& \left\|v-\left(I_{h, \Omega} v\right)^{\ell}\right\|_{L^{2}(\Omega)}+h\left\|v-\left(I_{h, \Omega} v\right)^{\ell}\right\|_{H^{1}(\Omega)} \leq C h^{r+1}\|v\|_{H^{r+1}(\Omega)}, \\
& \left\|\vartheta-\left(I_{h, \Gamma} \vartheta\right)^{\ell}\right\|_{L^{2}(\Gamma)}+h\left\|\vartheta-\left(I_{h, \Gamma} \vartheta\right)^{\ell}\right\|_{H^{1}(\Gamma)} \leq C h^{r+1}\|\vartheta\|_{H^{r+1}(\Gamma)},
\end{aligned}
$$

for all $v \in H^{r+1}(\Omega)$ and $\vartheta \in H^{r+1}(\Gamma)$ with $1 \leq r \leq p$; cf. [4, Prop. 5.4]. By construction, the nodes on the surface coincide with the bulk nodes, and therefore we have

$$
\gamma\left(I_{h, \Omega} v\right)=I_{h, \Gamma} \gamma(v) \quad \text { for all } v \in C(\bar{\Omega}) .
$$

The semidiscretized equation. As finite element space we choose $V_{h}=V_{h, p}^{\Omega}$. The discretizations $m_{h}, b_{h}, a_{h}: V_{h} \times V_{h} \rightarrow \mathbb{R}$ of $m, b$, and $a$ are defined via

$$
\begin{aligned}
m_{h}\left(v_{h}, \varphi_{h}\right):= & \int_{\Omega_{h}} v_{h} \varphi_{h} \mathrm{~d} \mathbf{x}+\int_{\Gamma_{h}} v_{h} \varphi_{h} \mathrm{~d} s, \\
b_{h}\left(v_{h}, \varphi_{h}\right):= & \int_{\Omega_{h}}\left(\left(I_{h, \Omega} \alpha_{\Omega}\right) v_{h}+\left(I_{h, \Omega} \beta_{\Omega}\right) \cdot \nabla v_{h}\right) \varphi_{h} \mathrm{~d} \mathbf{x} \\
& \quad+\int_{\Gamma_{h}}\left(\left(I_{h, \Gamma} \alpha_{\Gamma}\right) v_{h}+\left(I_{h, \Gamma} \beta_{\Gamma}\right) \cdot \nabla_{\Gamma} v_{h}\right) \varphi_{h} \mathrm{~d} s, \\
a_{h}\left(v_{h}, \varphi_{h}\right):= & \int_{\Omega_{h}} \nabla v_{h} \cdot \nabla \varphi_{h} \mathrm{~d} \mathbf{x}+\int_{\Gamma_{h}} \nabla_{\Gamma_{h}} u_{h} \cdot \nabla_{\Gamma_{h}} \varphi_{h} \mathrm{~d} s,
\end{aligned}
$$

and we discretize the nonlinearity $f_{h}:[0, T] \times V_{h} \rightarrow H_{h}$ via

$$
\begin{aligned}
m_{h}\left(f_{h}\left(t, v_{h}\right), \varphi_{h}\right):= & \int_{\Omega_{h}} I_{h, \Omega} \widetilde{f}_{\Omega}\left(t, \cdot, v_{h}^{\ell}(\cdot)\right)(\mathbf{x}) \varphi_{h}(\mathbf{x}) \mathrm{d} \mathbf{x} \\
& +\int_{\Gamma_{h}} I_{h, \Gamma} \widetilde{f}_{\Gamma}\left(t, \cdot, v_{h}^{\ell}(\cdot)\right)(\mathbf{x}) \varphi_{h}(\mathbf{x}) \mathrm{d} s
\end{aligned}
$$

for all $\varphi_{h} \in V_{h}$. 
REMARK 2.3. The nodal interpolation only requires function evaluations in the nodes $a_{1}, \ldots, a_{N}$. Since these are invariant under the lift operator, the computation of $v_{h}^{\ell}$ is not necessary. The lift is only needed for the definition of $f_{h}$ since the interpolation operator acts on functions on $\Omega$.

The discretized version of (2.7) is then given as a special case of

$$
\begin{aligned}
m_{h}\left(u_{h}^{\prime \prime}, \varphi_{h}\right)+b_{h}\left(u_{h}^{\prime}, \varphi_{h}\right)+a_{h}\left(u_{h}, \varphi_{h}\right) & =m_{h}\left(f_{h}\left(t, u_{h}, u_{h}^{\prime}\right), \varphi_{h}\right), \\
u_{h}(0)=u_{h}^{0}, \quad u_{h}^{\prime}(0) & =v_{h}^{0} .
\end{aligned}
$$

The discrete quantities then satisfy similar assumptions as their continuous counterparts:

ASSUMPTION 2.4.

(a) The bilinear form $a_{h}: V_{h} \times V_{h} \rightarrow \mathbb{R}$ is symmetric and there exists a constant $\widehat{c}_{G} \geq 0$ such that

$$
\widetilde{a}_{h}:=a_{h}+\widehat{c}_{G} m_{h}
$$

is a scalar product on $V_{h}$ with induced norm $\|\cdot\|_{\widetilde{a}_{h}}$.

(b) The bilinear form $m_{h}$ is also a scalar product on $V_{h}$. We denote $V_{h}$ equipped with this scalar product $m_{h}$ by $H_{h}$ and the induced norm by $\|\cdot\|_{m_{h}}$.

(c) The bilinear form $b_{h}: V_{h} \times H_{h} \rightarrow \mathbb{R}$ is bounded independent of $h$ and there exists $\widehat{\beta}_{\mathrm{qm}} \geq 0$ such that

$$
b_{h}\left(v_{h}, v_{h}\right)+\widehat{\beta}_{\mathrm{qm}}\left\|v_{h}\right\|_{m_{h}}^{2} \geq 0 \quad \text { for all } v_{h} \in V_{h} .
$$

(d) The nonlinearity $f_{h}:[0, T] \times V_{h} \times H_{h} \rightarrow H_{h}$ is locally Lipschitz-continuous on $V_{h} \times H_{h}$ with constant $\widehat{L}_{T, M}$.

(e) There exists a constant $\widehat{C}_{H, V}>0$ such that $\left\|v_{h}\right\|_{m_{h}} \leq \widehat{C}_{H, V}\left\|v_{h}\right\|_{\widetilde{a}_{h}}$ for all $v_{h} \in V_{h}$. All constants in this assumption should be independent of $h$.

REMARK 2.5. In a finite-dimensional space, all norms are equivalent. The crucial point in the last assumption is that the constants are independent of $h$, which corresponds to the continuous embedding $V \hookrightarrow H$.

In our specific example we have $\widehat{c}_{G}=1, \widehat{\beta}_{\mathrm{qm}}=0, \widehat{C}_{H, V}=1$; cf. [7]. The Lipschitzcontinuity of $f_{h}$ is proven in the following lemma.

LEMMA 2.6. The discretized nonlinearity $f_{h}:[0, T] \times V_{h} \rightarrow H_{h}$ defined in (2.11) is locally Lipschitz-continuous on $V_{h}$ with Lipschitz constant

$$
\widehat{L}_{T, M}=C\left(\sigma(\Omega)^{\frac{\zeta_{\Omega}-1}{2 \zeta_{\Omega}}}+\sigma(\Gamma)^{\frac{\zeta_{\Gamma}-1}{2 \zeta_{\Gamma}}}+2 M^{\zeta_{\Omega}-1}+2 M^{\zeta_{\Gamma}-1}\right),
$$

i.e., for all $u_{h}, v_{h} \in V_{h}$ with $\left\|u_{h}\right\|_{\widetilde{a}_{h}},\left\|v_{h}\right\|_{\widetilde{a}_{h}} \leq M$ and for all $t \in[0, T]$,

$$
\left\|f_{h}\left(t, u_{h}\right)-f_{h}\left(t, v_{h}\right)\right\|_{m_{h}} \leq \widehat{L}_{T, M}\left\|u_{h}-v_{h}\right\|_{\widetilde{a}_{h}} .
$$

The constant $C$ is independent of $h, \sigma(\Omega)$ and $\sigma(\Gamma)$ denote the measure of $\Omega$ and $\Gamma$, respectively, and $\zeta_{\Omega}$ and $\zeta_{\Gamma}$ are defined in (2.2).

Proof. Let $M>0, t<T$, and $u_{h}, v_{h} \in V_{h}$ such that $\left\|u_{h}\right\|_{\widetilde{a}_{h}},\left\|v_{h}\right\|_{\widetilde{a}_{h}}<M$. With the definition of $f_{h}$ in (2.11) and the Cauchy-Schwarz inequality, we obtain

$$
\begin{aligned}
\left\|f_{h}\left(t, u_{h}\right)-f_{h}\left(t, v_{h}\right)\right\|_{m_{h}}= & \sup _{\left\|\varphi_{h}\right\|_{m_{h}}=1} m_{h}\left(f_{h}\left(t, u_{h}\right)-f_{h}\left(t, v_{h}\right), \varphi_{h}\right) \\
\leq & \left\|I_{h, \Omega} \widetilde{f}_{\Omega}\left(t, \cdot, u_{h}^{\ell}(\cdot)\right)-I_{h, \Omega} \widetilde{f}_{\Omega}\left(t, \cdot, v_{h}^{\ell}(\cdot)\right)\right\|_{L^{2}\left(\Omega_{h}\right)} \\
& \quad+\left\|I_{h, \Gamma} \widetilde{f}_{\Gamma}\left(t, \cdot, u_{h}^{\ell}(\cdot)\right)-I_{h, \Gamma} \widetilde{f}_{\Gamma}\left(t, \cdot, v_{h}^{\ell}(\cdot)\right)\right\|_{L^{2}\left(\Gamma_{h}\right)} .
\end{aligned}
$$


In the following we derive a bound for the first term at the right-hand side; the second one can be bounded analogously.

This proof is more involved than the one for the continuous nonlinearity since the appearing interpolation operator is not continuous with respect to $L^{2}$. To work around this problem, we use discrete $L^{q}$-norms defined via

$$
\left\|v_{h}\right\|_{q}:=h^{\frac{d}{q}}\left(\sum_{i=1}^{N}\left|v_{h}\left(a_{i}\right)\right|^{q}\right)^{\frac{1}{q}} .
$$

Because of the scaling with $h^{\frac{d}{q}}$ and the mesh regularity, we have that the norm $\|\cdot\|_{q}$ is equivalent to $\|\cdot\|_{L^{q}\left(\Omega_{h}\right)}$ on $V_{h, p}^{\Omega}$ for all $q \in[2, \infty)$ with equivalence constants independent of $h$. This is well known for $q=2$. The generalization to $q \neq 2$ is straightforward; cf. [13, Lemma 5.2]. Combining this with the Sobolev embedding theorem (2.4) (with $\Omega_{h}$ instead of $\Omega)$, we have

$$
\left\|v_{h}\right\|_{2 \zeta_{\Omega}} \lesssim C\left\|v_{h}\right\|_{L^{2 \zeta_{\Omega}\left(\Omega_{h}\right)}} \lesssim C\left\|v_{h}\right\|_{H^{1}\left(\Omega_{h}\right)} \quad \text { for all } v_{h} \in V_{h},
$$

with constants $C$ independent of $h$.

The definition of the discrete norms, the growth conditions (2.3), and (2.13) yield

$$
\begin{aligned}
\| I_{h, \Omega} & \widetilde{f}_{\Omega}\left(t, \cdot, u_{h}^{\ell}(\cdot)\right)-I_{h, \Omega} \widetilde{f}_{\Omega}\left(t, \cdot, v_{h}^{\ell}(\cdot)\right) \|_{L^{2}\left(\Omega_{h}\right)}^{2} \\
& \leq C\left\|I_{h, \Omega} \widetilde{f}_{\Omega}\left(t, \cdot, u_{h}(\cdot)\right)-I_{h, \Omega} \widetilde{f}_{\Omega}\left(t, \cdot, v_{h}(\cdot)\right)\right\|_{2}^{2} \\
& =C h^{d}\left(\sum_{i=1}^{N}\left|\widetilde{f}_{\Omega}\left(t, a_{i}, u_{h}\left(a_{i}\right)\right)-\widetilde{f}_{\Omega}\left(t, a_{i}, v_{h}\left(a_{i}\right)\right)\right|^{2}\right) \\
& =C h^{d}\left(\sum_{i=1}^{N}\left|\left(u_{h}\left(a_{i}\right)-v_{h}\left(a_{i}\right)\right) \int_{0}^{1} \partial_{3} \widetilde{f}_{\Omega}\left(t, a_{i}, v_{h}\left(a_{i}\right)+\theta\left(u_{h}\left(a_{i}\right)-v_{h}\left(a_{i}\right)\right)\right) \mathrm{d} \theta\right|^{2}\right) \\
& \leq C h^{\frac{d\left(\zeta_{\Omega}-1\right)}{\zeta_{\Omega}}}\left(\sum_{i=1}^{N}\left(1+\left(\left|u_{h}\left(a_{i}\right)\right|+\left|v_{h}\left(a_{i}\right)\right|\right)^{\zeta_{\Omega}-1}\right)^{\frac{2 \zeta_{\Omega}}{\zeta_{\Omega}-1}}\right)^{\frac{\zeta_{\Omega}-1}{\zeta_{\Omega}}}\left\|u_{h}-v_{h}\right\|_{2 \zeta_{\Omega}}^{2} \\
& \leq C\left(\|1\|_{\frac{2 \zeta_{\Omega}}{\zeta_{\Omega}-1}}+\left\|u_{h}\right\|_{2 \zeta_{\Omega}}^{\zeta_{\Omega}-1}+\left\|v_{h}\right\|_{2 \zeta_{\Omega}}^{\zeta_{\Omega}-1}\right)^{2}\left\|u_{h}-v_{h}\right\|_{2 \zeta_{\Omega}}^{2} \\
& \leq C\left(\|1\|{ }_{L^{\frac{2 \zeta_{\Omega}}{\zeta_{\Omega}-1}}\left(\Omega_{h}\right)}+\left\|u_{h}\right\|_{L^{2} \zeta_{\Omega}\left(\Omega_{h}\right)}^{\zeta_{\Omega}-1}+\left\|v_{h}\right\|_{L^{2} \zeta_{\Omega}\left(\Omega_{h}\right)}^{\zeta_{\Omega}-1}\right)\left\|u_{h}-v_{h}\right\|_{H^{1}\left(\Omega_{h}\right)}^{2} \\
& \leq C\left(\sigma(\Omega)^{\frac{\zeta_{\Omega}-1}{2 \zeta_{\Omega}}}+2 M^{\zeta_{\Omega}-1}\right)\left\|u_{h}-v_{h}\right\|_{H^{1}\left(\Omega_{h}\right)}^{2},
\end{aligned}
$$

where we additionally used the bound

$$
\sigma\left(\Omega_{h}\right) \leq C \sigma(\Omega)
$$

which is satisfied independently of $h$.

2.3. Main result. We can now state the main result of the paper, namely the error bound for the bulk-surface discretization of wave equations with kinetic boundary conditions. The proof will be conducted in Section 4.

THEOREM 2.7. Let $\Gamma \in C^{p+1}, \alpha_{\Omega} \in H^{p}(\bar{\Omega}), \beta_{\Omega} \in H^{p}(\bar{\Omega})^{d}, \alpha_{\Gamma} \in H^{p}(\Gamma)$, and $\beta_{\Gamma} \in$ $H^{p}(\Gamma)^{d}$. Furthermore, let $u$ be a solution of $(2.1)$ on $[0, T]$ with 
(a) $u \in C^{2}\left([0, T] ; H^{2}(\Omega ; \Gamma)\right) \cap L^{\infty}\left([0, T] ; H^{\max \{4, p+2\}}(\Omega ; \Gamma)\right)$,

(b) $u^{\prime} \in L^{\infty}\left([0, T] ; H^{p+1}(\Omega ; \Gamma)\right)$, and

(c) $u^{\prime \prime} \in L^{\infty}\left([0, T] ; H^{p}(\Omega ; \Gamma)\right)$.

Then there exist $h^{*}, M>0$ such that for all $h<h^{*}$, the solution $u_{h}$ of (2.12) exists on $[0, T]$ and satisfies the error bound

$$
\left\|u_{h}^{\ell}(t)-u(t)\right\|_{H^{1}(\Omega ; \Gamma)}+\left\|\left(u_{h}^{\prime}\right)^{\ell}(t)-u^{\prime}(t)\right\|_{L^{2}(\Omega) \times L^{2}(\Gamma)} \leq C \mathrm{e}^{\left(\widehat{L}_{T, M}+\frac{1}{2}\right) t}(1+t) h^{p}
$$

with $\widehat{L}_{T, M}$ from Lemma 2.6 and a constant $C$ independent of $h$ and $t$.

3. Unified error analysis (UEA) for nonconforming discretizations. In this section we present the UEA for a general class of nonconforming space discretizations of semilinear wave equations. The UEA is a tool that provides a priori error bounds in terms of interpolation, data, and conformity errors of the method. These bounds can be used to derive convergence rates for a large class of problems in a simple, systematic, and modular way. The idea is to treat wave equations abstractly as evolution equations in Hilbert spaces and their space discretizations as differential equations in finite-dimensional Hilbert spaces and to perform the error analysis in this abstract setting.

Here we briefly recall the setting used in $[7,8]$ and extend it to the semilinear case. As in [8], we start by proving an error bound for discretizations of first-order evolution equations in Section 3.1 and then use this result to prove error bounds for second-order equations in Section 3.2. This result will then be used in the next section to prove Theorem 2.7. More applications of the unified error analysis can be found in [8].

3.1. Semilinear evolution equations with monotone operators. We start by stating an abstract evolution equation and introduce a general space discretization afterwards.

The continuous problem. Let $X$ be a Hilbert space with scalar product $p$. We consider the evolution equation

$$
\begin{aligned}
x^{\prime}(t)+S x(t) & =g(t, x(t)), \quad t \in(0, T], \\
x(0) & =x^{0} .
\end{aligned}
$$

ASSUMPTION 3.1.

(a) The linear operator $S: D(S) \rightarrow X$ is the generator of a $C_{0}$-semigroup with

$$
\left\|\mathrm{e}^{-t S}\right\|_{X \leftarrow X} \leq \mathrm{e}^{c_{\mathrm{qm}} t}
$$

(b) The nonlinearity $g \in C^{1}([0, T] \times X ; X)$ is locally Lipschitz continuous with respect to the second component with constant $L_{T, M}$.

The following classical well-posedness result can be found in [15] for example.

LEMMA 3.2. If Assumption 3.1 holds true, then (3.1) is locally well-posed, i.e., for every $x^{0} \in X$ there exists $t^{*}\left(x^{0}\right)>0$ such that for all $T<t^{*}\left(x^{0}\right)$, (3.1) has a unique solution

$$
x \in C^{1}([0, T] ; X) \cap C([0, T] ; D(S)) .
$$

Abstract space discretization. We consider a general space discretization of (3.1) and provide an abstract error result for a large class of equations and discretizations.

Let $X_{h}$ be a finite-dimensional Hilbert space with scalar product $p_{h}$. In this space we seek the numerical approximation $x_{h}$. Moreover, let $S_{h} \in \mathcal{L}\left(X_{h}, X_{h}\right)$ and $g_{h}:[0, T] \times X_{h} \rightarrow X_{h}$ be discretizations of $S$ and $g$, respectively. Similar to their continuous counterparts, we 


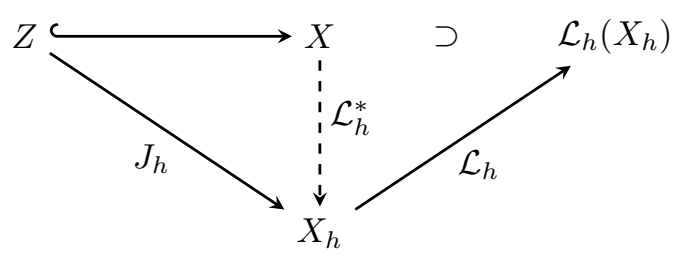

FIG. 3.1. Overview of spaces and operators; $c f$. [8].

require that $S_{h}$ and $g_{h}$ satisfy Assumption 3.1 with $X_{h}$ instead of $X$ and constants $\widehat{c}_{\mathrm{qm}}, \widehat{L}_{T, M}$ independent of $h$.

Then the discretized version of the evolution equation (3.1) is given by

$$
\begin{aligned}
x_{h}^{\prime}(t)+S_{h} x_{h}(t) & =g_{h}\left(t, x_{h}(t)\right), \quad t \in(0, T], \\
x_{h}(0) & =x_{h}^{0} .
\end{aligned}
$$

Due to the Picard-Lindelöf theorem, (3.3) is locally well-posed, and we denote the maximal existence time of the solution by $t_{h}^{*}\left(x_{h}^{0}\right)$.

Error analysis. Our framework allows us to treat nonconforming space discretizations, where $X_{h} \nsubseteq X$. To relate the continuous and discrete quantities, we therefore assume that there exists a lift operator $\mathcal{L}_{h}: X_{h} \rightarrow X$ that satisfies

$$
\left\|\mathcal{L}_{h} y_{h}\right\|_{X} \leq C_{X}\left\|y_{h}\right\|_{X_{h}} \quad \text { for all } y_{h} \in X_{h}
$$

with $C_{X}$ independent of $h$. We then define the lifted discrete space

$$
X_{h}^{\ell}:=\mathcal{L}_{h}\left(X_{h}\right) \subset X .
$$

Let $\mathcal{L}_{h}^{*}: X \rightarrow X_{h}$ be the adjoint of the lift operator, i.e.,

$$
p_{h}\left(\mathcal{L}_{h}^{*} y, y_{h}\right)=p\left(y, \mathcal{L}_{h} y_{h}\right) \quad \text { for all } y \in X, y_{h} \in X_{h} .
$$

Furthermore, for a Hilbert space $Z$, which is densely and continuously embedded in $X$, we make use of a reference operator $J_{h} \in \mathcal{L}\left(Z, X_{h}\right)$ satisfying

$$
\left\|J_{h}\right\|_{X_{h} \leftarrow Z} \leq C_{J}
$$

with a constant $C_{J}$ independent of $h$. The reference operator should satisfy $\mathcal{L}_{h} J_{h} z \approx z$ for all $z \in Z$ and could, e.g., be an interpolation or a projection operator. Figure 3.1 illustrates the operators between the spaces.

Finally, we define the linear remainder operator

$$
R_{h}:=\mathcal{L}_{h}^{*} S-S_{h} J_{h}: D(S) \cap Z \rightarrow X_{h}
$$

and the nonlinear remainder operator $r_{h}:[0, T] \times Z \rightarrow X_{h}$ via

$$
r_{h}(t, z):=\mathcal{L}_{h}^{*} g(t, z)-g_{h}\left(t, J_{h} z\right) .
$$

If the solution of the discretized equation (3.3) is bounded, then we can state an error bound in terms of the approximation errors of $x^{0}, x$ and $x^{\prime}$, and of the remainder operators $R_{h}, r_{h}$. We make the following regularity assumption on the continuous solution: 
Assumption 3.3. The solution $x$ of (3.1) satisfies $x \in C^{1}\left(\left[0, t^{*}\left(x^{0}\right)\right) ; Z\right)$.

THEOREM 3.4. Let Assumption 3.3 be satisfied, $T<\min \left\{t^{*}\left(x^{0}\right), t_{h}^{*}\left(x_{h}^{0}\right)\right\}$, and

$$
M_{h}=\max \left\{C_{J}\|x\|_{L^{\infty}([0, T] ; Z)},\left\|x_{h}\right\|_{L^{\infty}\left([0, T] ; X_{h}\right)}\right\} .
$$

Then, for all $t \in[0, T]$, the lifted discrete solution of (3.3) satisfies the error bound

$$
\left\|\mathcal{L}_{h} x_{h}(t)-x(t)\right\|_{X} \leq C \mathrm{e}^{\left(\widehat{L}_{T, M_{h}}+\widehat{C}_{\mathrm{qm}}\right) t} E_{h}(t)+\left\|\left(\mathrm{I}-\mathcal{L}_{h} J_{h}\right) x(t)\right\|_{X}
$$

with

$$
\begin{aligned}
E_{h}(t)=\| & x_{h}^{0}-J_{h} x^{0}\left\|_{X_{h}}+t\right\|\left(\mathcal{L}_{h}^{*}-J_{h}\right) x^{\prime} \|_{L^{\infty}\left([0, T] ; X_{h}\right)} \\
& +t\left\|R_{h} x\right\|_{L^{\infty}\left([0, T] ; X_{h}\right)}+t\left\|r_{h}(\cdot, x(\cdot))\right\|_{L^{\infty}\left([0, T] ; X_{h}\right)} .
\end{aligned}
$$

Proof. The proof consists of four steps.

(I) Splitting of the error: We split the error into

$$
\mathcal{L}_{h} x_{h}-x=\mathcal{L}_{h} e_{h}+e_{J_{h}}
$$

with the discrete error

$$
e_{h}=x_{h}-J_{h} x \in X_{h}
$$

and the reference error

$$
e_{J_{h}}=\left(\mathcal{L}_{h} J_{h}-\mathrm{I}\right) x \text {. }
$$

This splitting yields

$$
\left\|\mathcal{L}_{h} x_{h}-x\right\|_{X} \leq C_{X}\left\|e_{h}\right\|_{X_{h}}+\left\|\left(\mathcal{L}_{h} J_{h}-\mathrm{I}\right) x\right\|_{X},
$$

where the last summand only depends on the choice of the reference and the lift operator.

(II) Derivation of an evolution equation for the error $e_{h}$ : Since $x \in C^{1}([0, T], Z)$ and $J_{h} \in \mathcal{L}\left(Z, X_{h}\right)$, we have $e_{h} \in C^{1}\left([0, T] ; X_{h}\right)$ and

$$
e_{h}^{\prime}=x_{h}^{\prime}-J_{h} x^{\prime}=\left(x_{h}^{\prime}-\mathcal{L}_{h}^{*} x^{\prime}\right)+\left(\mathcal{L}_{h}^{*}-J_{h}\right) x^{\prime} .
$$

Using the continuous and the discrete equations (3.1) and (3.3), we can rewrite the first term on the right-hand side as

$$
\begin{aligned}
x_{h}^{\prime}-\mathcal{L}_{h}^{*} x^{\prime} & =-S_{h} x_{h}+g_{h}\left(\cdot, x_{h}\right)-\mathcal{L}_{h}^{*}(-S x+g(\cdot, x)) \\
& =-S_{h} e_{h}+g_{h}\left(\cdot, x_{h}\right)-\mathcal{L}_{h}^{*} g(\cdot, x)+\left(\mathcal{L}_{h}^{*} S-S_{h} J_{h}\right) x .
\end{aligned}
$$

So, we end up with the following equation for the discrete error:

$$
\begin{aligned}
e_{h}^{\prime}+S_{h} e_{h} & =\left(\mathcal{L}_{h}^{*}-J_{h}\right) x^{\prime}+R_{h} x+g_{h}\left(\cdot, x_{h}\right)-\mathcal{L}_{h}^{*} g(\cdot, x) \\
& =\left(\mathcal{L}_{h}^{*}-J_{h}\right) x^{\prime}+R_{h} x-r_{h}(\cdot, x)+g_{h}\left(\cdot, x_{h}\right)-g_{h}\left(\cdot, J_{h} x\right) \\
& =: d_{h} .
\end{aligned}
$$

Hence, $e_{h}$ satisfies a linear evolution equation in $X_{h}$. 
(III) Stability: By the variation-of-constants formula, we have

$$
\begin{aligned}
\left\|e_{h}(t)\right\|_{X_{h}} & \leq\left\|\mathrm{e}^{-t S_{h}} e_{h}(0)\right\|_{X_{h}}+\int_{0}^{t}\left\|\mathrm{e}^{-(t-s) S_{h}} d_{h}(s)\right\|_{X_{h}} \mathrm{~d} s \\
& \leq \mathrm{e}^{\widehat{c}_{\mathrm{qm}} t}\left\|e_{h}(0)\right\|_{X_{h}}+\mathrm{e}^{\widehat{c}_{\mathrm{gm}} t} \int_{0}^{t} \mathrm{e}^{-\widehat{c}_{\mathrm{qm}} s}\left\|d_{h}(s)\right\|_{X_{h}} \mathrm{~d} s .
\end{aligned}
$$

Using the Lipschitz-continuity of $g_{h}$ and the definition of the nonlinear remainder (3.6), we are able to bound the defect $d_{h}$ by

$$
\begin{aligned}
\left\|d_{h}(s)\right\|_{X_{h}} \leq\left\|\left(\mathcal{L}_{h}^{*}-J_{h}\right) x^{\prime}(s)\right\|_{X_{h}} & +\left\|R_{h} x(s)\right\|_{X_{h}} \\
& +\left\|r_{h}(s, x(s))\right\|_{X_{h}}+\widehat{L}_{T, M_{h}}\left\|e_{h}(s)\right\|_{X_{h}} .
\end{aligned}
$$

(IV) Abstract error estimate: Inserting (3.10) into (3.9) yields

$$
\begin{aligned}
\mathrm{e}^{-\widehat{c}_{\mathrm{qm}} t}\left\|e_{h}(t)\right\|_{X_{h}} \leq & \left\|e_{h}(0)\right\|_{X_{h}}+t\left\|\left(\mathcal{L}_{h}^{*}-J_{h}\right) x^{\prime}\right\|_{L^{\infty}\left([0, T] ; X_{h}\right)}+t\left\|R_{h} x\right\|_{L^{\infty}\left([0, T] ; X_{h}\right)} \\
& +t\left\|r_{h}(\cdot, x(\cdot))\right\|_{L^{\infty}\left([0, T] ; X_{h}\right)}+\widehat{L}_{T, M_{h}} \int_{0}^{t} \mathrm{e}^{-\widehat{c}_{\mathrm{qm} m} s}\left\|e_{h}(s)\right\|_{X_{h}} \mathrm{~d} s \\
= & E_{h}(t)+\widehat{L}_{T, M_{h}} \int_{0}^{t} \mathrm{e}^{-\widehat{c}_{\mathrm{qm}} s}\left\|e_{h}(s)\right\|_{X_{h}} \mathrm{~d} s
\end{aligned}
$$

With the Grönwall lemma we finally obtain

$$
\left\|e_{h}(t)\right\|_{X_{h}} \leq \mathrm{e}^{\left(\widehat{L}_{T, M_{h}}+\widehat{c}_{\mathrm{qm}}\right) t} E_{h}(t) .
$$

Together with (3.8) this proves the error bound (3.7).

The following corollary shows, under additional consistency assumptions, that the discretized equation remains bounded for sufficiently small $h$ and that the discrete solution converges to the continuous one.

Corollary 3.5. Let Assumption 3.3 be satisfied and $T<t^{*}\left(x^{0}\right)$. Moreover, assume that

$$
\lim _{h \rightarrow 0} E_{h}(t) \rightarrow 0 \quad \text { for all } t \in[0, T] .
$$

Then there exists $h^{*}>0$, such that $x_{h}$ exists for all $h<h^{*}$ on $[0, T]$ with

$$
\left\|x_{h}\right\|_{L^{\infty}\left([0, T] ; X_{h}\right)} \leq M:=2 C_{J}\|x\|_{L^{\infty}([0, T] ; Z)} .
$$

Furthermore, the error bound (3.7) holds true with $M_{h}=M$.

If additionally

$$
\lim _{h \rightarrow 0}\left\|\left(\mathrm{I}-\mathcal{L}_{h} J_{h}\right) x(t)\right\|_{X} \rightarrow 0 \quad \text { for all } t \in[0, T]
$$

holds true, then the lifted numerical solution converges, i.e.,

$$
\left\|\mathcal{L}_{h} x_{h}(t)-x(t)\right\|_{X} \stackrel{h \rightarrow 0}{\longrightarrow} 0, \quad t \in[0, T] .
$$

Proof. We only have to show that $x_{h}$ exists for all $h<h^{*}$ on $[0, T]$ with

$$
\left\|x_{h}\right\|_{L^{\infty}\left([0, T] ; X_{h}\right)} \leq M
$$


The other assertions then follow immediately from Theorem 3.4.

We define

$$
T_{h}:=\sup \left\{t \in\left(0, t_{h}^{*}\left(x_{h}^{0}\right)\right) \mid\left\|x_{h}\right\|_{L^{\infty}\left([0, t] ; X_{h}\right)} \leq M\right\}
$$

as the maximal time for which the discrete solution stays bounded by $M$. Clearly, we have $T_{h}<t_{h}^{*}\left(x_{h}^{0}\right)$ and further, by Theorem 3.4, for all $t \leq \min \left\{T, T_{h}\right\}$, we have

$$
\begin{aligned}
\left\|x_{h}(t)\right\|_{X_{h}} & \leq\left\|x_{h}(t)-J_{h} x(t)\right\|_{X_{h}}+\left\|J_{h} x(t)\right\|_{X_{h}} \\
& \leq\left\|e_{h}(t)\right\|_{X_{h}}+\frac{M}{2} \\
& \leq C \mathrm{e}^{\left(\widehat{L}_{T, M}+\widehat{c}_{\mathrm{qm}}\right) t} E_{h}(t)+\frac{M}{2} \stackrel{h \rightarrow 0}{\longrightarrow} \frac{M}{2} .
\end{aligned}
$$

Hence, there exists an $h^{*}>0$ such that $\left\|x_{h}(t)\right\|_{X_{h}} \leq \frac{3}{4} M$ for all $h<h^{*}$ and $t \leq \min \left\{T, T_{h}\right\}$. Since $x_{h}$ is continuous and by the definition of $T_{h}$, we thus get

$$
t_{h}^{*}\left(x_{h}^{0}\right)>T_{h}>T \quad \text { and } \quad\left\|x_{h}\right\|_{L^{\infty}\left([0, T] ; X_{h}\right)}<M
$$

for all $h<h^{*}$.

3.2. Second-order semilinear wave-type equations. Next, we apply the results of Section 3.1 to general second-order wave equations. Again, we start by stating the framework.

The continuous problem. Let $V, H$ be Hilbert spaces and let $V$ be densely embedded in $H$. We consider the variational differential equation (2.7) as a prototype for weak formulations of second-order wave equations and assume that Assumption 2.2 holds true. By the dense embedding of the Hilbert spaces, there exists a constant $C_{H, V}>0$ such that

$$
\|v\|_{m} \leq C_{H, V}\|v\|_{\widetilde{a}} \quad \text { for all } v \in V .
$$

In order to reformulate the problem as an evolution equation on $H$, we define the operators $A: D(A) \rightarrow H$ and $B: V \rightarrow H$ corresponding to $a$ and $b$ via

$$
\begin{array}{ll}
m(A v, w)=a(v, w), & \text { for all } v \in D(A), w \in V, \\
m(B v, w)=b(v, w), & \text { for all } v \in V, w \in H,
\end{array}
$$

with

$$
D(A)=\left\{v \in V \mid \exists C=C(v)>0 \text { such that } \forall w \in V:|a(v, w)| \leq C\|w\|_{m}\right\} .
$$

Equation (2.7) then reads: Find $u \in C^{2}([0, T] ; H) \cap C^{1}([0, T] ; V) \cap C([0, T] ; D(A))$ such that

$$
u^{\prime \prime}(t)+B u^{\prime}(t)+A u(t)=f\left(t, u(t), u^{\prime}(t)\right), \quad u(0)=u^{0}, \quad u^{\prime}(0)=v^{0} .
$$

By construction, a solution of (3.11) is also a solution of (2.7).

First-order formulation. To analyze the well-posedness and the space discretizations of (3.11), we want to apply the theory of Section 3.1 and therefore rewrite (3.11) as a first-order equation. Let $u^{\prime}=v$ and define

$$
x=\left[\begin{array}{l}
u \\
v
\end{array}\right], \quad S=\left[\begin{array}{cc}
0 & -\mathrm{I} \\
A & B
\end{array}\right], \quad g(t, x)=\left[\begin{array}{c}
0 \\
f(t, u, v)
\end{array}\right], \quad x^{0}=\left[\begin{array}{l}
u^{0} \\
v^{0}
\end{array}\right] .
$$


The linear operator $S$ is defined on its domain $D(S)=D(A) \times V$. With $X=V \times H$, (3.11) is equivalent to (3.1).

LEMMA 3.6. The operator $-S$ is the generator of a $C_{0}$-semigroup on $X=V \times H$ which satisfies (3.2) with constant $c_{\mathrm{qm}}=\frac{1}{2} c_{G} C_{H, V}+\beta_{\mathrm{qm}}$.

Proof. This follows from a combination of Lemma 4.2 (with $\alpha=1$ ), Lemma 2.3, and Theorem 2.4 in [8].

Since $f \in C^{1}([0, T] \times V \times H ; H)$ implies $g \in C^{1}([0, T] \times X ; X)$, the problem (3.11) is locally well-posed by Lemma 3.2. We denote the maximal existence time by $t^{*}\left(u^{0}, v^{0}\right)$.

Space discretization. Let $V_{h}$ be a finite-dimensional vector space. We consider (2.12) as a space discretization of (2.7) and assume that Assumption 2.4 is satisfied.

To reformulate (2.12) as an evolution equation we define $A_{h}, B_{h} \in \mathcal{L}\left(V_{h} ; V_{h}\right)$ via

$$
m_{h}\left(A_{h} v_{h}, \varphi_{h}\right)=a_{h}\left(v_{h}, \varphi_{h}\right), \quad m_{h}\left(B_{h} v_{h}, \varphi_{h}\right)=b_{h}\left(v_{h}, \varphi_{h}\right) \quad \text { for all } v_{h}, \varphi_{h} \in V_{h} .
$$

Then, (2.12) is equivalent to

$$
\begin{aligned}
u_{h}^{\prime \prime}(t)+B_{h} u_{h}^{\prime}(t)+A_{h} u_{h}(t) & =f_{h}\left(t, u_{h}(t), u_{h}^{\prime}(t)\right), \\
u_{h}(0)=u_{h}^{0}, \quad u_{h}^{\prime}(0) & =v_{h}^{0} .
\end{aligned}
$$

Analogously to the continuous case, we can rewrite this as a first-order equation. With the Hilbert space $X_{h}=V_{h} \times H_{h}$ and

$$
x_{h}(t)=\left[\begin{array}{l}
u_{h}(t) \\
v_{h}(t)
\end{array}\right], \quad S_{h}=\left[\begin{array}{cc}
0 & -\mathrm{I} \\
A_{h} & B_{h}
\end{array}\right], \quad g_{h}\left(t, x_{h}(t)\right)=\left[\begin{array}{c}
0 \\
f_{h}\left(t, u_{h}(t), v_{h}(t)\right)
\end{array}\right],
$$

(3.12) has the form (3.3). Similarly to Lemma 3.6, we obtain that $-S_{h}$ is the generator of a $C_{0}$-semigroup on $X_{h}$ which satisfies (3.2) with constant $\widehat{c}_{\mathrm{qm}}=\frac{1}{2} \widehat{c}_{G} \widehat{C}_{H, V}+\widehat{\beta}_{\mathrm{qm}}$ independent of $h$.

Due to the Picard-Lindelöf theorem, (3.12) is locally well-posed, and we denote the maximal existence time of the solution by $t_{h}^{*}\left(u_{h}^{0}, v_{h}^{0}\right)$.

Error analysis. To apply the error result from Section 3.1 we have to specify the operators occurring there.

We assume that there exists a lift operator $\mathcal{L}_{h}^{V} \in \mathcal{L}\left(V_{h} ; V\right)$ satisfying

$$
\left\|\mathcal{L}_{h}^{V} v_{h}\right\|_{m} \leq C_{H}\left\|v_{h}\right\|_{m_{h}}, \quad\left\|\mathcal{L}_{h}^{V} v_{h}\right\|_{\widetilde{a}} \leq C_{V}\left\|v_{h}\right\|_{\widetilde{a}_{h}}
$$

for all $v_{h} \in V_{h}$ with constants $C_{H}, C_{V}>0$ independent of $h$. Using this, we define the lift operator $\mathcal{L}_{h}: X_{h} \rightarrow X$ by

$$
\mathcal{L}_{h}\left[\begin{array}{c}
v_{h} \\
w_{h}
\end{array}\right]:=\left[\begin{array}{c}
\mathcal{L}_{h}^{V} v_{h} \\
\mathcal{L}_{h}^{V} w_{h}
\end{array}\right] .
$$

Note that one lift operator $\mathcal{L}_{h}^{V}$ is sufficient since $V \hookrightarrow H$, but we have to distinguish the adjoints $\mathcal{L}_{h}^{V^{*}}: V \rightarrow V_{h}$ and $\mathcal{L}_{h}^{H *}: H \rightarrow H_{h}$ with respect to the scalar products in $V$ and $H$. They are defined via

$$
\begin{aligned}
m_{h}\left(\mathcal{L}_{h}^{H *} v, w_{h}\right) & =m\left(v, \mathcal{L}_{h}^{V} w_{h}\right) & & \text { for all } v \in H, w_{h} \in H_{h}, \\
\widetilde{a}_{h}\left(\mathcal{L}_{h}^{V *} v, w_{h}\right) & =\widetilde{a}\left(v, \mathcal{L}_{h}^{V} w_{h}\right) & & \text { for all } v \in V, w_{h} \in V_{h} .
\end{aligned}
$$

Let $Z^{V} \stackrel{d}{\hookrightarrow} V$ be a subspace of $V$ and $I_{h} \in \mathcal{L}\left(Z^{V} ; V_{h}\right)$ be an interpolation operator satisfying

$$
\left\|I_{h}\right\|_{H_{h} \leftarrow Z^{V}} \leq C_{I}
$$


with $C_{I}>0$ independent of $h$. We define the first-order reference operator $J_{h}: Z \rightarrow X_{h}$ by

$$
J_{h}\left[\begin{array}{c}
v \\
w
\end{array}\right]:=\left[\begin{array}{c}
\mathcal{L}_{h}^{V *} v \\
I_{h} w
\end{array}\right]
$$

on $Z=V \times Z^{V} \stackrel{d}{\hookrightarrow} X$.

REMARK 3.7. We used $I_{h}$ instead of $\mathcal{L}_{h}^{H *}$ in the second component of the reference operator because the adjoint lift operator only leads to suboptimal error bounds.

By (3.13) and (3.14), conditions (3.4) and (3.5) are satisfied with $C_{X}=\max \left\{C_{V}, C_{H}\right\}$ and $C_{J}=\max \left\{C_{V}, C_{I}\right\}$.

For $v_{h}, w_{h} \in V_{h}$, the errors in the scalar products are defined via

$$
\begin{aligned}
\Delta m\left(v_{h}, w_{h}\right) & :=m\left(\mathcal{L}_{h}^{V} v_{h}, \mathcal{L}_{h}^{V} w_{h}\right)-m_{h}\left(v_{h}, w_{h}\right), \\
\Delta \widetilde{a}\left(v_{h}, w_{h}\right) & :=\widetilde{a}\left(\mathcal{L}_{h}^{V} v_{h}, \mathcal{L}_{h}^{V} w_{h}\right)-\widetilde{a}_{h}\left(v_{h}, w_{h}\right),
\end{aligned}
$$

and, for $z=(u, v) \in Z$, the linear and nonlinear remainder terms are given by

$$
\begin{gathered}
R_{h} z=\left(\mathcal{L}_{h}^{*} S-S_{h} J_{h}\right) z=\left[\begin{array}{c}
-\left(\mathcal{L}_{h}^{V *}-I_{h}\right) v \\
\mathcal{L}_{h}^{H *}(A u+B v)-\left(A_{h} \mathcal{L}_{h}^{V *} u+B_{h} I_{h} v\right)
\end{array}\right], \\
r_{h}(t, z)=\mathcal{L}_{h}^{*} g(t, z)-g_{h}\left(t, J_{h} z\right)=\left[\begin{array}{c}
0 \\
\mathcal{L}_{h}^{H *} f(t, u, v)-f_{h}\left(t, \mathcal{L}_{h}^{V *} u, I_{h} v\right)
\end{array}\right],
\end{gathered}
$$

respectively.

To obtain an error bound for the semi discretization from Theorem 3.4 we have to bound the remainder terms. The nonlinear one is obviously bounded by

$$
\left\|r_{h}(t, z)\right\|_{X_{h}}=\left\|\mathcal{L}_{h}^{H *} f(t, u, v)-f_{h}\left(t, \mathcal{L}_{h}^{V *} u, I_{h} v\right)\right\|_{m_{h}}, \quad z=(u, v) \in Z .
$$

For the linear one we get

$$
\begin{aligned}
\left\|R_{h}\left[\begin{array}{l}
u \\
v
\end{array}\right]\right\|_{X_{h}} \leq & C \max _{\left\|\varphi_{h}\right\|_{\tilde{a}_{h}}=1}\left|\Delta \widetilde{a}\left(I_{h} v, \varphi_{h}\right)\right|+\max _{\left\|\varphi_{h}\right\|_{\tilde{a}_{h}}=1}\left|\Delta \widetilde{a}\left(I_{h} u, \varphi_{h}\right)\right| \\
& +\max _{\left\|\psi_{h}\right\|_{m_{h}}=1}\left|\Delta m\left(I_{h} u, \psi_{h}\right)\right|+\left\|\left(\mathrm{I}-\mathcal{L}_{h}^{V} I_{h}\right) u\right\|_{\widetilde{a}} \\
& \left.+\left\|\left(\mathrm{I}-\mathcal{L}_{h}^{V} I_{h}\right) v\right\|_{\widetilde{a}}+\max _{\left\|\psi_{h}\right\|_{m_{h}}=1}\left|b\left(v, \mathcal{L}_{h}^{V} \psi_{h}\right)-b_{h}\left(I_{h} v, \psi_{h}\right)\right|\right),
\end{aligned}
$$

i.e., it can be bounded by errors in the bilinear forms and interpolation errors. The bound (3.16) is proven in Lemma 4.7 in [8] (with our choice of the reference operator $J_{h}$ ). Proving the final error bound requires a sufficiently regular solution.

ASSUMPTION 3.8. The solution $u$ of (3.11) satisfies $u \in C^{2}\left(\left[0, t^{*}\left(u^{0}, v^{0}\right)\right) ; Z^{V}\right)$.

The following two results are direct consequences of Theorem 3.4 and Corollary 3.5.

TheOREM 3.9. Let Assumption 3.8 be satisfied and $T<\min \left\{t^{*}\left(u^{0}, v^{0}\right), t_{h}^{*}\left(u_{h}^{0}, v_{h}^{0}\right)\right\}$. Then, for all $t \in[0, T]$, the lifted semidiscrete solution $\mathcal{L}_{h}^{V} u_{h}$ of (3.12) satisfies the error bound

$$
\left\|\mathcal{L}_{h}^{V} u_{h}(t)-u(t)\right\|_{\widetilde{a}}+\left\|\mathcal{L}_{h}^{V} u_{h}^{\prime}(t)-u^{\prime}(t)\right\|_{m} \leq C \mathrm{e}^{\left(\widehat{L}_{T, M_{h}}+\widehat{c}_{q m}\right) t}(1+t) \sum_{i=1}^{5} E_{i}
$$


with a constant $C$ that is independent of $h$ and $t$. The other constants are given by $\widehat{c}_{\mathrm{qm}}=\frac{1}{2} \widehat{c}_{G} \widehat{C}_{H, V}+\widehat{\beta}_{\mathrm{qm}}$,

$$
M_{h}=\max \left\{\max \left\{C_{V}, C_{I}\right\}\left\|\left[\begin{array}{c}
u \\
u^{\prime}
\end{array}\right]\right\|_{L^{\infty}\left([0, T] ; V \times Z^{V}\right)},\left\|\left[\begin{array}{c}
u_{h} \\
u_{h}^{\prime}
\end{array}\right]\right\|_{L^{\infty}\left([0, T] ; V_{h} \times H_{h}\right)}\right\},
$$

and

$$
\begin{aligned}
E_{1}:= & \left\|u_{h}^{0}-\mathcal{L}_{h}^{V *} u^{0}\right\|_{\widetilde{a}_{h}}+\left\|v_{h}^{0}-I_{h} v^{0}\right\|_{m_{h}}, \\
E_{2}:= & \left\|\mathcal{L}_{h}^{H *} f\left(\cdot, u(\cdot), u^{\prime}(\cdot)\right)-f_{h}\left(\cdot, \mathcal{L}_{h}^{V *} u(\cdot), I_{h} u^{\prime}(\cdot)\right)\right\|_{L^{\infty}\left([0, T] ; H_{h}\right)}, \\
E_{3}:= & \left\|\left(\mathrm{I}-\mathcal{L}_{h}^{V} I_{h}\right) u\right\|_{L^{\infty}([0, T] ; V)}+\left\|\left(\mathrm{I}-\mathcal{L}_{h}^{V} I_{h}\right) u^{\prime}\right\|_{L^{\infty}([0, T] ; V)} \\
& +\left\|\left(\mathrm{I}-\mathcal{L}_{h}^{V} I_{h}\right) u^{\prime \prime}\right\|_{L^{\infty}([0, T] ; H)}, \\
E_{4}:= & \left\|_{\left\|\varphi_{h}\right\|_{\tilde{a}_{h}}=1} \Delta \widetilde{a}\left(I_{h} u, \varphi_{h}\right)\right\|_{L^{\infty}(0, t)}+\|\|_{\left\|\psi_{h}\right\|_{m_{h}}=1} \Delta m\left(I_{h} u, \psi_{h}\right) \|_{L^{\infty}(0, t)} \\
& +\left\|_{\left\|\varphi_{h}\right\|_{\tilde{a}_{h}}=1} \Delta \widetilde{a}\left(I_{h} u^{\prime}, \varphi_{h}\right)\right\|_{L^{\infty}(0, t)}+\left\|_{\left\|\psi_{h}\right\|_{m_{h}}=1} \Delta m\left(I_{h} u^{\prime \prime}, \psi_{h}\right)\right\|_{L^{\infty}(0, t)}, \\
E_{5}:= & \left\|_{\left\|\psi_{h}\right\|_{m_{h}}=1} \mid b\left(u^{\prime}, \mathcal{L}_{h}^{V} \psi_{h}\right)-b_{h}\left(I_{h} u^{\prime}, \psi_{h}\right)\right\| \|_{L^{\infty}(0, t)} .
\end{aligned}
$$

If $E_{i} \rightarrow 0, i=1, \ldots, 5$, then we can conclude convergence.

COROllary 3.10. Let Assumption 3.8 be satisfied, $T<t^{*}\left(u^{0}, v^{0}\right)$, and

$$
M:=2 \max \left\{C_{V}, C_{I}\right\}\left\|\left[\begin{array}{c}
u \\
u^{\prime}
\end{array}\right]\right\|_{L^{\infty}\left([0, T] ; V \times Z^{V}\right)} .
$$

Further, let $E_{i} \stackrel{h \rightarrow 0}{\longrightarrow} 0$ for $i=1, \ldots, 5$. Then there exists $h^{*}>0$, such that $u_{h}$ exists in $[0, T]$ for all $h<h^{*}$ with

$$
\left\|\left[\begin{array}{l}
u_{h} \\
u_{h}^{\prime}
\end{array}\right]\right\|_{L^{\infty}\left([0, T] ; V_{h} \times H_{h}\right)} \leq M .
$$

Additionally, the error bound (3.17) holds true with $M_{h}=M$, and the lifted semidiscrete solution converges, i.e.,

$$
\lim _{h \rightarrow 0}\left\|\mathcal{L}_{h}^{V} u_{h}(t)-u(t)\right\|_{\widetilde{a}}+\left\|\mathcal{L}_{h}^{V} u_{h}^{\prime}(t)-u^{\prime}(t)\right\|_{m}=0, \quad t \in[0, T] .
$$

Proof of Theorem 3.9. We apply Theorem 3.4. Recall that we have

$$
C_{X}=\max \left\{C_{V}, C_{H}\right\}, \quad C_{J}=\max \left\{C_{V}, C_{I}\right\}, \quad \widehat{c}_{\mathrm{qm}}=\frac{1}{2} \widehat{c}_{G} \widehat{C}_{H, V}+\widehat{\beta}_{\mathrm{qm}} .
$$

As in the proof of Theorem 4.8 in [8], we obtain (3.17) by applying the error estimate (3.7) and using (3.15) and (3.16).

Corollary 3.10 follows directly from Corollary 3.5. 
4. Proof of Theorem 2.7. For the proof we use the results of Section 3.2.

Proof of Theorem 2.7. We already showed in Section 2 that the weak formulation of the wave equations with kinetic boundary conditions (2.7) as well as their discretizations with the bulk-surface FEM fit into the general setting presented in Section 3.2.

We define the space

$$
Z^{V}:=H^{2}(\Omega ; \Gamma) \stackrel{d}{\hookrightarrow} V=H^{1}(\Omega ; \Gamma),
$$

the interpolation operator $I_{h}:=I_{h, \Omega}$, and the lift operator via

$$
\mathcal{L}_{h}^{V} v:=v^{\ell}
$$

with $v^{\ell}$ given in (2.8). By (2.9) we have $\mathcal{L}_{h} \in \mathcal{L}\left(V_{h} ; V\right)$. Moreover, (3.13) is satisfied and $I_{h}$ satisfies (3.14) by (2.10).

Hence, all assumptions of Corollary 3.10 are satisfied. It remains to bound the error terms by $\mathcal{O}\left(h^{p}\right)$ to obtain the desired error bound. In [7, Theorem 7.4] it was shown that

$$
E_{1}, E_{3}, E_{4}, E_{5} \leq C h^{p},
$$

so that we only have to study the nonlinear error term. By Lemma 2.6 we have

$$
\begin{aligned}
E_{2} & =\left\|\mathcal{L}_{h}^{H *} f(\cdot, u)-f_{h}\left(\cdot, \mathcal{L}_{h}^{V *} u\right)\right\|_{L^{\infty}\left([0, T] ; H_{h}\right)} \\
& \leq\left\|\mathcal{L}_{h}^{H *} f(\cdot, u)-f_{h}\left(\cdot, I_{h} u\right)\right\|_{L^{\infty}\left([0, T] ; H_{h}\right)}+\left\|f_{h}\left(\cdot, I_{h} u\right)-f_{h}\left(\cdot, \mathcal{L}_{h}^{V *} u\right)\right\|_{L^{\infty}\left([0, T] ; H_{h}\right)} \\
& \leq\left\|\mathcal{L}_{h}^{H *} f(\cdot, u)-f_{h}\left(\cdot, I_{h} u\right)\right\|_{L^{\infty}\left([0, T] ; H_{h}\right)}+\widehat{L}_{T, M}\left\|\left(I_{h}-\mathcal{L}_{h}^{V *}\right) u\right\|_{L^{\infty}\left([0, T] ; H_{h}\right)} .
\end{aligned}
$$

The second summand is of order $h^{p+1}$, and for the first one, by definition of $f$ and $f_{h}$, we obtain

$$
\begin{aligned}
& \left\|\mathcal{L}_{h}^{H *} f(t, u)-f_{h}\left(t, I_{h} u\right)\right\|_{m_{h}} \\
= & \sup _{\left\|\varphi_{h}\right\|_{m_{h}}=1} m_{h}\left(\mathcal{L}_{h}^{H *} f(t, u)-f_{h}\left(t, I_{h} u\right), \varphi_{h}\right) \\
= & \sup _{\left\|\varphi_{h}\right\|_{m_{h}}=1}\left(m\left(f(t, u), \mathcal{L}_{h}^{V} \varphi_{h}\right)-m_{h}\left(f_{h}\left(t, I_{h} u\right), \varphi_{h}\right)\right) \\
= & \sup _{\left\|\varphi_{h}\right\|_{m_{h}}=1}\left(\int_{\Omega} \widetilde{f}_{\Omega}(t, \mathbf{x}, u(\mathbf{x})) \varphi_{h}^{\ell}(\mathbf{x}) \mathrm{d} \mathbf{x}-\int_{\Omega_{h}} I_{h, \Omega} \widetilde{f}_{\Omega}\left(t, \cdot,\left(I_{h, \Omega} u\right)^{\ell}(\cdot)\right)(\mathbf{x}) \varphi_{h}(\mathbf{x}) \mathrm{d} \mathbf{x}\right. \\
& \left.+\int_{\Gamma} \widetilde{f}_{\Gamma}(t, \mathbf{x}, \gamma(u)(\mathbf{x})) \varphi_{h}^{\ell}(\mathbf{x}) \mathrm{d} s-\int_{\Gamma_{h}} I_{h, \Gamma} \widetilde{f}_{\Gamma}\left(t, \cdot,\left(I_{h, \Gamma} \gamma(u)\right)^{\ell}(\cdot)\right)(\mathbf{x}) \varphi_{h}(\mathbf{x}) \mathrm{d} s\right) .
\end{aligned}
$$

Let $\varphi_{h} \in V_{h}$ with $\left\|\varphi_{h}\right\|_{m_{h}}=1$. For the error in $\Omega$ we obtain

$$
\begin{aligned}
\int_{\Omega} \widetilde{f}_{\Omega}(t, \mathbf{x}, u(\mathbf{x})) \varphi_{h}^{\ell}(\mathbf{x}) \mathrm{d} \mathbf{x}-\int_{\Omega_{h}} I_{h, \Omega} \widetilde{f}_{\Omega}\left(t, \cdot,\left(I_{h, \Omega} u\right)^{\ell}(\cdot)\right)(\mathbf{x}) \varphi_{h}(\mathbf{x}) \mathrm{d} \mathbf{x} \\
=\int_{\Omega} \widetilde{f}_{\Omega}(t, \mathbf{x}, u(\mathbf{x})) \varphi_{h}^{\ell}(\mathbf{x}) \mathrm{d} \mathbf{x}-\int_{\Omega_{h}} I_{h, \Omega} \widetilde{f}_{\Omega}(t, \cdot, u(\cdot))(\mathbf{x}) \varphi_{h}(\mathbf{x}) \mathrm{d} \mathbf{x} \\
=\int_{\Omega} \widetilde{f}_{\Omega}(t, \mathbf{x}, u(\mathbf{x})) \varphi_{h}^{\ell}(\mathbf{x}) \mathrm{d} \mathbf{x}-\int_{\Omega}\left(I_{h, \Omega} \widetilde{f}_{\Omega}(t, \cdot, u(\cdot))\right)^{\ell}(\mathbf{x}) \varphi_{h}^{\ell}(\mathbf{x}) \mathrm{d} \mathbf{x} \\
\quad+\int_{\Omega}\left(I_{h, \Omega} \widetilde{f}_{\Omega}(t, \cdot, u(\cdot))\right)^{\ell}(\mathbf{x}) \varphi_{h}^{\ell}(\mathbf{x}) \mathrm{d} \mathbf{x}-\int_{\Omega_{h}} I_{h, \Omega} \widetilde{f}_{\Omega}(t, \cdot, u(\cdot))(\mathbf{x}) \varphi_{h}(\mathbf{x}) \mathrm{d} \mathbf{x}
\end{aligned}
$$


where we used the definition of the nodal interpolation in the first step, which tells us that the inner interpolation can be omitted since the outer interpolation only depends on the function values at the nodes $a_{i}$ which are invariant under the inner interpolation.

For the first term, with (2.9), (2.10), and $\left\|\varphi_{h}\right\|_{L^{2}(\Omega)} \leq\left\|\varphi_{h}\right\|_{m_{h}}=1$, we obtain

$$
\begin{aligned}
& \int_{\Omega} \widetilde{f}_{\Omega}(t, \mathbf{x}, u(\mathbf{x})) \varphi_{h}^{\ell}(\mathbf{x}) \mathrm{d} \mathbf{x}-\int_{\Omega}\left(I_{h, \Omega} \tilde{f}_{\Omega}(t, \cdot, u(\cdot))\right)^{\ell}(\mathbf{x}) \varphi_{h}^{\ell}(\mathbf{x}) \mathrm{d} \mathbf{x} \\
& \leq\left\|\widetilde{f}_{\Omega}(t, \cdot, u(\cdot))-\left(I_{h, \Omega} \widetilde{f}_{\Omega}(t, \cdot, u(\cdot))\right)^{\ell}\right\|_{L^{2}(\Omega)}\left\|\varphi_{h}^{\ell}\right\|_{L^{2}(\Omega)} \\
& \leq C_{\Omega, \Omega_{h}} C h^{p}\left\|\tilde{f}_{\Omega}(t, \cdot, u(\cdot))\right\|_{H^{p}(\Omega)} \\
& \leq C h^{p}\left(\left\|u_{t t}\right\|_{H^{p}(\Omega)}+\left\|\nabla u_{t}\right\|_{H^{p}(\Omega)}+\|\Delta u\|_{H^{p}(\Omega)}\right) .
\end{aligned}
$$

In the last step we used the differential equation (2.1).

Since $I_{h, \Omega} \widetilde{f}_{\Omega}(t, \cdot, u(\cdot)) \in V_{h, p}^{\Omega}$, we can bound the second term with the estimate (5.10) from [8] by

$$
\begin{aligned}
\int_{\Omega}\left(I_{h, \Omega} \widetilde{f}_{\Omega}(t, \cdot, u(\cdot))\right)^{\ell}(\mathbf{x}) \varphi_{h}^{\ell}(\mathbf{x}) & \mathrm{d} \mathbf{x}-\int_{\Omega_{h}} I_{h, \Omega} \widetilde{f}_{\Omega}(t, \cdot, u(\cdot))(\mathbf{x}) \varphi_{h}(\mathbf{x}) \mathrm{d} \mathbf{x} \\
& \leq C h^{p}\left\|I_{h, \Omega} \widetilde{f}_{\Omega}(t, \cdot, u(\cdot))\right\|_{L^{2}\left(\Omega_{h}\right)}\left\|\varphi_{h}\right\|_{L^{2}\left(\Omega_{h}\right)} \\
& \leq C h^{p}\left\|\widetilde{f}_{\Omega}(t, \cdot, u(\cdot))\right\|_{H^{2}(\Omega)} \\
& \leq C h^{p}\left(\left\|u_{t t}\right\|_{H^{2}(\Omega)}+\left\|\nabla u_{t}\right\|_{H^{2}(\Omega)}+\|\Delta u\|_{H^{2}(\Omega)}\right) .
\end{aligned}
$$

Here we also used $I_{h, \Omega} \in \mathcal{L}\left(H^{2}(\Omega) ; L^{2}\left(\Omega_{h}\right)\right)$ and the differential equation (2.1).

The error term on $\Gamma$ can be bounded analogously, and we obtain

$$
E_{2} \leq C h^{p}\left(\left\|u^{\prime \prime}(t)\right\|_{H^{p}(\Omega ; \Gamma)}+\|u(t)\|_{H^{\max \{4, p+2\}}(\Omega ; \Gamma)}\right) .
$$

The only additional term that has to be bounded in this case is $\left\|\partial_{n} u\right\|_{H^{p}(\Omega)}$, for which we get

$$
\left\|\partial_{n} u\right\|_{H^{p}(\Omega)} \leq\|u\|_{H^{p+2}(\Omega)} .
$$

This completes the proof.

5. Numerical examples. In this section we illustrate Theorem 2.7 numerically. We choose $\Omega=B(0,1) \subset \mathbb{R}^{2}$ as the two-dimensional unit sphere and

$$
u(t, \mathbf{x})=\mathrm{e}^{-t} \mathbf{x}_{1}^{2} \mathbf{x}_{2}^{2} .
$$

Furthermore, we set

$$
\begin{aligned}
& \eta_{\Omega}(t, \mathbf{x})=-\left(4+\mathrm{e}^{-t} \mathbf{x}_{1}^{2} \mathbf{x}_{2}^{2}\right) \mathrm{e}^{-t} \mathbf{x}_{1}^{2} \mathbf{x}_{2}^{2}-2 \mathrm{e}^{-t}\left(\mathbf{x}_{1}^{2}+\mathbf{x}_{2}^{2}\right), \\
& \eta_{\Gamma}(t, \mathbf{x})=\left(21-\mathrm{e}^{-2 t} \mathbf{x}_{1}^{4} \mathbf{x}_{2}^{4}\right) \mathrm{e}^{-t} \mathbf{x}_{1}^{2} \mathbf{x}_{2}^{2}-2 \mathrm{e}^{-t}
\end{aligned}
$$

Then, $u$ solves the semilinear wave equation with kinetic boundary conditions

$$
\begin{aligned}
u_{t t}+(1+\mathbf{x} \cdot \nabla) u_{t}-\Delta u & =|u| u+\eta_{\Omega}(t, \mathbf{x}), & (0, T) \times \Omega, \\
u_{t t}+\partial_{n} u-\Delta_{\Gamma} u & =|u|^{2} u+\eta_{\Gamma}(t, \mathbf{x}), & (0, T) \times \partial \Omega, \\
u(0, \mathbf{x}) & =0, \quad u_{t}(0, \mathbf{x})=2 \pi \mathbf{x}_{1} \mathbf{x}_{2}, & \text { in } \bar{\Omega} .
\end{aligned}
$$


We implemented the bulk-surface FEM by using the $\mathrm{C}++$ finite element-library deal.II $[2,3]$ with discrete initial values $u_{h}^{0}=I_{h, \Omega} u^{0}$ and $v_{h}^{0}=I_{h, \Omega} v^{0}$. For time integration, we applied the Crank-Nicolson scheme with sufficiently small step size such that the time integration error is negligible. The codes are available from the authors on request.

In Figure 5.1 the error

$$
\left\|u_{h}(t)-\left.u(t)\right|_{\Omega_{h}}\right\|_{H^{1}\left(\Omega_{h} ; \Gamma_{h}\right)}+\left\|u_{h}^{\prime}(t)-\left.u^{\prime}(t)\right|_{\Omega_{h}}\right\|_{L^{2}\left(\Omega_{h}\right) \times L^{2}\left(\Gamma_{h}\right)}
$$

is plotted against the mesh width $h$ for the discretization of the test example with isoparametric elements of order $p=1$ and $p=2$ at $t=0.8$. We evaluated the integrals with a quadrature rule of degree $2 p$, so that the quadrature error is negligible. The restriction of $u$ to $\Omega_{h}$ is possible since $\Omega_{h} \subset \Omega$, for convex domains.

The error behaves as predicted by Theorem 2.7.

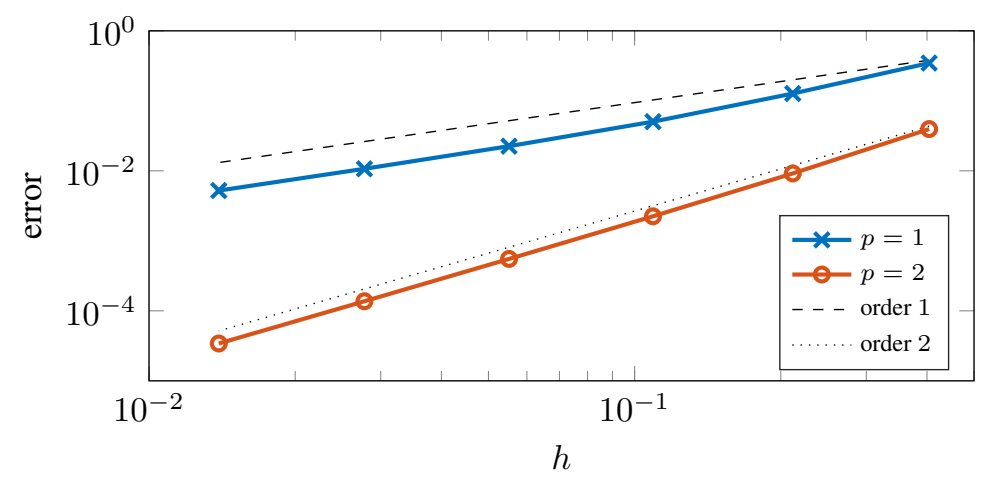

FIG. 5.1. Error (5.1) at $t=0.8$ for the test example.

Funding. Funded by the Deutsche Forschungsgemeinschaft (DFG, German Research Foundation) - Project-ID 258734477 - SFB 1173.

Acknowledgments. We thank Ralf Hiptmair for the fruitful discussions about the proof of Lemma 2.6 and David Hipp for many inspiring conversations.

\section{REFERENCES}

[1] R. A. Adams And J. J. F. Fournier, Sobolev Spaces, 2nd ed., Academic Press, Amsterdam, 2003.

[2] G. Alzetta, D. Arndt, W. Bangerth, V. Boddu, B. Brands, D. Davydov, R. Gassmoeller, T. Heister, L. Heltai, K. Kormann, M. Kronbichler, M. Maier, J.-P. Pelteret, B. TurCKSIN, AND D. WELls, The deal.II library, version 9.0, J. Numer. Math., 26 (2018), pp. 173-183.

[3] W. BANGERTH, R. HARTMANN, AND G. KANSCHAT, deal.II - a general purpose object oriented finite element library, ACM Trans. Math. Software, 33 (2007), Art. 24, 27 pages.

[4] C. M. ElLIOTT AND T. RANNER, Finite element analysis for a coupled bulk-surface partial differential equation, IMA J. Numer. Anal., 33 (2013), pp. 377-402.

[5] H. Goldberg, W. KAMPOWSKy, AND F. TRÖLTZSCH, On Nemytskij operators in $L_{p}$-spaces of abstract functions, Math. Nachr., 155 (1992), pp. 127-140.

[6] P. GRISVARD, Elliptic Problems in Nonsmooth Domains, SIAM, Philadelphia, 2011.

[7] D. HiPP, A Unified Error Analysis for Spatial Discretizations of Wave-Type Equations with Applications to Dynamic Boundary Conditions, PhD. Thesis, Institut für Angewandte und Numerische Mathematik, Karlsruhe Institute of Technology, Karlsruhe, 2017.

[8] D. HIPP, M. HOCHBRUCK, AND C. STOHRER, Unified error analysis for nonconforming space discretizations of wave-type equations, IMA J. Numer. Anal., 39 (2019), pp. 1206-1245. 
[9] D. HIPP AND B. KovÁCS, Finite element error analysis of wave equations with dynamic boundary conditions: L2 estimates, IMA J. Numer. Anal., drz073, 2020.

https://doi.org/10.1093/imanum/drz073

[10] M. HochBrUCK, B. MAIER, AND C. STOHRER, Heterogeneous multiscale method for Maxwell's equations, Multiscale Model. Simul., 17 (2019), pp. 1147-1171.

[11] T. Kashiwabara, C. M. Colciago, L. Dedè, AND A. Quarteroni, Well-posedness, regularity, and convergence analysis of the finite element approximation of a generalized Robin boundary value problem, SIAM J. Numer. Anal., 53 (2015), pp. 105-126.

[12] B. KovÁCS AND C. LUBICH, Numerical analysis of parabolic problems with dynamic boundary conditions, IMA J. Numer. Anal., 37 (2017), pp. 1-39.

[13] J. LeIBOLD, Semilineare Wellengleichungen mit Dynamischen Randbedingungen, Master Thesis, Fakultät für Mathematik, Karlsruhe Institute of Technology, Karlsruhe, 2017.

[14] S. NICAISE, Convergence and stability analyses of hierarchic models of dissipative second order evolution equations, Collect. Math., 68 (2017), pp. 433-462.

[15] A. PAZY, Semigroups of Linear Operators and Applications to Partial Differential Equations, Springer, New York, 1983

[16] E. VitilLARO, Strong solutions for the wave equation with a kinetic boundary condition, in Recent Trends in Nonlinear Partial Differential Equations. I. Evolution Problems, J. B. Serrin, E. L. Mitidieri, and V. D. Rădulescu, eds., vol. 594 of Contemp. Math., Amer. Math. Soc., Providence, 2013, pp. 295-307.

[17] - On the wave equation with hyperbolic dynamical boundary conditions, interior and boundary damping and source, Arch. Ration. Mech. Anal., 223 (2017), pp. 1183-1237. 